\title{
The Proof of the Riemann Hypothesis on a Relativistic Turing Machine
}

\author{
Yuriy N. Zayko \\ Department of Applied Informatics, Faculty of Public Administration, The Russian Presidential Academy of National Economy and Public \\ Administration, Saratov, Russia \\ Email address: \\ zyrnick@rambler.ru

\section{To cite this article:} \\ Yuriy N. Zayko. The Proof of the Riemann Hypothesis on a Relativistic Turing Machine. International Journal of Theoretical and Applied \\ Mathematics. Vol. 3, No. 6, 2017, pp. 219-224. doi: 10.11648/j.ijtam.20170306.17
}

Received: October 2, 2017; Accepted: November 13, 2017; Published: January 2, 2018

\begin{abstract}
In this article, the proof of the Riemann hypothesis is considered using the calculation of the Riemann $\zeta$-function on a relativistic computer. The work lies at the junction of the direction known as "Beyond Turing", considering the application of the so-called "relativistic supercomputers" for solving non-computable problems and a direction devoted to the study of non-trivial zeros of the Riemann $\zeta$-function. Considerations are given in favor of the validity of the Riemann hypothesis with respect to the distribution of non-trivial zeros of the $\zeta$-function.
\end{abstract}

Keywords: Metric, Riemann $\zeta$-Function, Non-Computable Problems, Singularity, Black Hole, Relativistic Computer, Riemann Hypothesis, Beyond Turing

\section{Introduction}

In the article [1] a relativistic Turing machine was suggested for calculation the Riemann $\zeta$-function presented by the series which diverges for $u<1$ [2].

$$
\zeta(w)=\sum_{n=1}^{\infty} n^{-w}, w=u+i v
$$

Such an approach (known as "Beyond Turing") to solving problems beyond the range of problems solved by the classical Turing machine develops in a number of works since the 1980s of the last century (see, for example, [3]). The corresponding computing devices received a name of "relativistic supercomputers". An unchanging attribute of the proposed projects is black holes, as the sources of the metric required for their implementation. The approach developed below, so as in the author's previous articles [1, 4], is free from this necessity. The calculation of the sum of a divergent series (a non-computable problem) is regarded as a physical problem about the motion of a material point in a space-time which curvature is due to the calculation itself. One can imagine a relativistic Turing machine, the role of the head of which is performed by a material particle moving in accordance with relativistic equations of motion determined by the corresponding physical problem. In the article [1] the computations are performed for the critical line $u=1 / 2$. Below are presented the calculations both in and outside it. Considerations are given in favor of the validity of the Riemann hypothesis with respect to the distribution of nontrivial zeros of the $\zeta$-function.

\section{Calculation the $\zeta$-Function in the Critical Line}

In the article [1] was investigated the behavior in the complex plane of partial sums of the divergent series, representing the Riemann $\zeta$-function (hereinafter, the $\zeta$ function)

$$
\zeta_{m}(w)=\sum_{n=1}^{m} n^{-w}
$$

where $n, m$ are natural numbers, $w=u+i v$ is a complex argument of the $\zeta$-function. As was shown in [1] the behavior of (1) resembles the motion of a particle along the trajectory of a vortex for sufficiently large $m$. This physical problem defines the relativistic Turing machine used in this paper. 
In the article [1] were received the expression for the interval $d s$ and the relativistic equations of motion of the material particle moving along the vortex trajectory in its own frame of references

$$
\begin{aligned}
& d s^{2}=A(r)(c d t)^{2}-d l^{2}+B(r) d r^{2}, \\
& A(r)=b^{2}-\left(\frac{\tilde{r}}{r}\right)^{2}, b^{2}=1-\left(\frac{\delta}{\omega}\right)^{2}, \\
& B(r)=\left\{1-\left(\frac{r}{\tilde{r}}\right)^{2}-\left(\frac{\delta}{\omega} \frac{r}{\tilde{r}}+\frac{\tilde{r}}{r_{\varphi}^{\prime}}\right)^{2} A^{-1}\right\}
\end{aligned}
$$

where we set $\tilde{r}=\omega / c, c$ is a speed of light. Here are used the asymptotic expressions for the radial and angle components of the speed of a particle which realizes the calculation

$$
V_{\varphi}=\omega / r, V_{r}=\delta / r
$$

( $\omega, \delta$ - are constants) for sufficiently large $r$ in the polar coordinate system $r, \phi$ on the complex plane.

Besides that, we use instead an angle element $d \phi$ the length element along the vortex trajectory $d l$, which is defined as

$$
\begin{aligned}
& d l^{2}=d r^{2}+r^{2} d \varphi^{2} \\
& r_{\varphi}^{\prime}=\frac{ \pm r r_{s}^{\prime}}{\sqrt{1-\left(r_{s}^{\prime}\right)^{2}}}, r_{s}^{\prime}=\frac{d r}{d s}
\end{aligned}
$$

The last equation in (4) will be used in the subsequent numerical calculations. In these variables, the equations of motion look as follows [1]

$$
\begin{aligned}
& \frac{d^{2} x^{0}}{d s^{2}}+A^{-1} A_{r}^{\prime} \frac{d x^{0}}{d s} \frac{d r}{d s}=0, x^{0}=c t, \\
& \frac{d^{2} l}{d s^{2}}=0, \\
& \frac{d^{2} r}{d s^{2}}-\frac{A_{r}^{\prime}}{2 B}\left(\frac{d x^{0}}{d s}\right)^{2}+\frac{B_{r}^{\prime}}{2 B}\left(\frac{d r}{d s}\right)^{2}=0 ;
\end{aligned}
$$

The solutions of these equations received in [1] describe the behavior of the particle above the horizon $r_{0}=\tilde{r} / b$, which corresponds $A(r)=0$. Integrating the last equation in (5) one can receive the equation [1]

$$
y=\left(\frac{d r}{d s}\right)^{2}=\left[C_{2}-\frac{b^{4}}{A(r)}\right] \frac{1}{B(r)}
$$

$C_{2}$-is a constant.

To describe the behavior the particle under the horizon one must define a new metric which has no a fictitious singularity at $r=r_{0}$. It is more convenient to use instead coordinates $r, \phi$ which are used in [1] coordinates $r, l$ introduced earlier.

To construct a metric suitable for $r<r_{0}$, following [5], we perform the transformation of coordinates $r, t \rightarrow \rho, \tau^{1}$.

1 A similar method was firstly applied by D. Finkelstein [5]

$$
d r=\frac{f \cdot(d \rho \pm c d \tau)}{\sqrt{-A(r) B(r)}}, c d t=\frac{f^{2} d \rho \pm c d \tau}{1-f^{2}}
$$

where $f(r)$-is a function chosen later from the condition that the fictitious singularity at $r=r_{0}$ of the metric (2) be eliminated. Signs \pm correspond to the motion in and out of the field center $r=0$. Performing the calculations, we find that this is achieved by choosing

$$
f(r)=[1-A(r)]^{1 / 2}=\left[\left(\frac{\delta}{\omega}\right)^{2}+\left(\frac{\tilde{r}}{r}\right)^{2}\right]^{1 / 2}
$$

The expression for the interval (2) takes the form

$$
d s^{2}=(c d \tau)^{2}-f^{2}(r) d \rho^{2}-d l^{2}
$$

The interval (9) has only the real singularity at the point $r$ $=0$. The metric (9) is synchronous $\left(g_{\tau \tau}=1\right)$ and nonstationary, as in the Schwarzschild case [5]. From (7) it follows the connection between the new and old coordinates

$$
\begin{aligned}
& \rho \pm c \tau=\Phi(r), \\
& \Phi(r)=\int \frac{\sqrt{-A(r) B(r)}}{f(r)} d r
\end{aligned}
$$

In variables $\rho, \tau$, there is no singularity on the horizon $r=\Phi^{-1}(\rho-c \tau)=r_{0}$. The coordinate $\rho$ is everywhere spatial and $\tau$-temporary (some clarifications are given below). The given values of $r$ correspond to the world lines $\rho-c \tau=$ const . The world lines of a particle at rest relative to the reference frame described by coordinates $\rho, \tau$ are straight lines parallel to the axis $\tau$.

As shown in the article [1] the above reasoning describes the behavior of the $\zeta$-function on the critical line $u=1 / 2$. It contains both nontrivial zeros of the $\zeta$-function and points that are not its zeros. For zeros $w=w_{z}$, moving the particle along the world line in a finite interval of proper time ends at the center of the field $r=0$. For other points $w=w_{n z}$ that are not zeros of the $\zeta$-function, the particle, moving along the world line, ends its approach to the center $r=0$ at a point $r=\left|\zeta\left(w_{n z}\right)\right|$. The argument $\zeta\left(w_{n z}\right)$ is found based the considerations, which is given below.

Using the methods of general theory of relativity we write out the system of equations of motion in the region below the horizon

$$
\begin{aligned}
& \frac{d^{2} \tau}{d s^{2}}=0, \\
& \frac{d^{2} l}{d s^{2}}=0, \\
& \frac{d^{2} \rho}{d s^{2}}+\frac{1}{f} \frac{d f}{d \rho}\left(\frac{d \rho}{d s}\right)^{2}+\frac{2}{c f} \frac{\partial f}{\partial \tau} \frac{d \rho}{d s}=0
\end{aligned}
$$

where the expressions for the non-zero Christoffel symbols are used [5]. 
Note the contradictoriness of the transformations (7) and the followed expressions for the interval (9) and equations (11). Indeed, on the one hand, the coordinate system $\rho, \tau$ is synchronous, since the components of the metric tensor $g_{\alpha 0}=$ $0(\alpha=1, \rho)$ [5]. On the other hand, it is impossible to synchronize watches of observers located on different sides of the horizon, since they can not exchange light signals. Therefore, strictly speaking, we can use transformations (7) and formulas (9) and (11) only above the horizon. Usually, this is sentenced to the fact that Finkelstein's coordinate system, in turn, is incomplete, which is overcome by introducing another, complete system, for example, KruskalSzekeres [5]. As shown below, the incompleteness of the Finkelstein reference system is due to fundamental physical causes and can not be eliminated in this way.

After the particle crossed the horizon, another system should be used to describe its motion

$$
\begin{aligned}
& \frac{d^{2} l}{d s^{2}}=0 \\
& \frac{d^{2} \rho}{d s^{2}}+\frac{1}{f} \frac{d f}{d \rho}\left(\frac{d \rho}{d s}\right)^{2}=0
\end{aligned}
$$

which doesn't contain the time $\tau$. Below is a more complete justification for excluding the time $\tau$ from the description.

From the equation (12) and (10) we find

$$
\frac{d r}{d s}= \pm \frac{C_{3}}{\sqrt{-A(r) B(r)}}
$$

$C_{3}$ is a constant. Bearing in mind that the solution of (13) must be sewn on the horizon with a similar solution (6), which is valid over the horizon we find $C_{3}=b^{2}$.

Equations (6) and (13) can be integrated. The results of the numerical calculation are shown in Figure 1

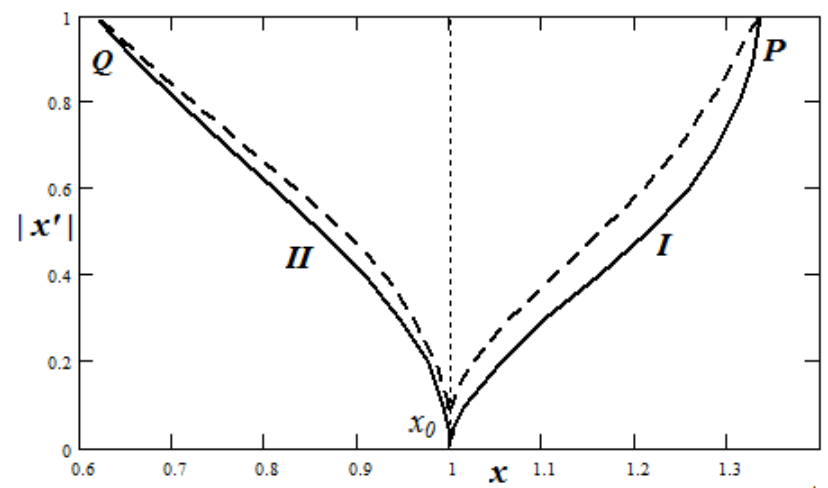

Figure 1. The phase portrait of the solutions of equations (6) (I) and (13) (II). The solid and the dashed lines show the two branches of the solution, dotted line-the horizon $x_{0} ; P, Q$-are the branch points, where $r_{s}^{\prime}= \pm 1$.

$I: x=r / \tilde{r}>x_{0}, x^{\prime}=d r / d s ; C_{2}=1,5 ; I I: x=r / \tilde{r}<x_{0} ; x_{0}=1,001$.
The qualitative behavior of the solution can be described as follows. In the moving system, the point representing the behavior of the particle placed above the horizon begins to move along the branch, for which $r_{s}^{\prime}>0$ corresponding to an increase the helix radius over time. In the branch point $P$ $r_{s}^{\prime}=1$, what corresponds $\left(r_{\varphi}^{\prime}\right)^{2} \rightarrow \infty$ or $d \varphi \rightarrow 0$, i.e. rotation is stopped. Then the particle goes to another branch, for which $r_{s}^{\prime}<0$ and, moving along this branch and rotating in the opposite direction, reaches the horizon $r=r_{0}$. More detailed picture of particle motion will be given below.

Further, the particle will continue to move under the horizon. The particle continues its movement to another branching point $Q$, where $\left|r_{s}^{\prime}\right|=1$ too and changes the direction of its movement on the opposite one. After that, another cycle of periodic motion begins.

For the data in Figure 1, the horizon $x_{0}=r_{0} / \tilde{r}=1.001$. On the horizon, the radial velocity of the particle can be found from the equation

$$
\frac{d r\left(r_{0}\right)}{d s}=\frac{ \pm b^{2}}{\frac{\delta}{\omega b}+\frac{\tilde{r}}{r_{\varphi}^{\prime}\left(r_{0}\right)}}
$$

The \pm signs correspond to two branches of the solution, shown in Figure 1. Solving equation (14) with allowance for (4) we obtain the value $r_{s}^{\prime}\left(r_{0}\right) \approx \pm \delta / \omega$ in which the higher order terms with respect to the small parameter $\delta / \omega<<1$ were omitted.

This scenario is typical for the argument $w$ of the $\zeta$ function, which corresponds to its non-zero value $w \neq w_{n z}$. Therefore, the particle will not fall to the center of the field $r$ $=0$, but only reach the branch point $Q$, whose character is the same as for the point $P$ in Figure 1. The argument $\zeta(w)$ is determined by the angle $\phi$ at which branching takes place, i.e. $r_{s}^{\prime}= \pm 1$ in the formula (4).

A point chosen arbitrarily on the critical line will almost always not be a zero of the $\zeta$-function: $w \neq w_{n z}$. Therefore, the choice of the conditions under which equation (13) is solved does not affect the nature of the solution.

Consider the calculation of the $\zeta$-function for an argument which coincides with its zero: $w=w_{z}$. The trajectory of the particle realizing this calculation belongs to the special trajectories of equations (13). Along it, the angle $\phi$ does not change, i.e. $d \phi=0$, and $d l= \pm d r$, and the relation (4) ceases to work. Nevertheless, the solution (13) obtained can be used by putting in it $r_{\varphi}^{\prime}=\infty$. The result is shown in Figure 2 . 


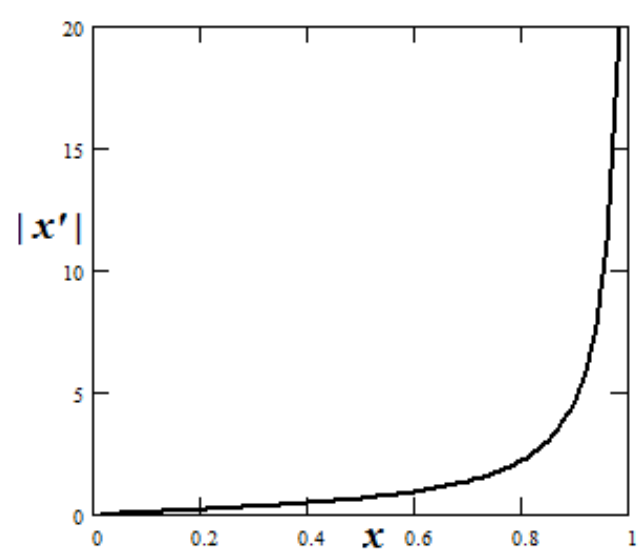

Figure 2. The phase portrait of the solution of equation (13) for computing the zero of the $\zeta$-function. The notation and data are the same as in Figure 1: $x=r / \tilde{r}<x_{0}=1.001, x^{\prime}=d r / d s$.

\section{Calculation of the $\zeta$-Function Outside the Critical Line ${ }^{2}$}

Since the details of calculating the $\zeta$-function outside the critical line in many respects repeat its computation on the critical line, we omit many of them and confine ourselves mainly to the study of the special trajectories of the equations of motion under the horizon that are related to the zeros of the $\zeta$-function. As for the case of the critical line, the motion of the particle realizing computation resembles the motion along the trajectory of a vortex. In a fixed coordinate system $r^{\prime}, \varphi^{\prime}, z^{\prime}, t^{\prime}$ (we use cylindrical spatial coordinates) the interval looks as follows

$$
\begin{aligned}
& d s^{\prime 2}=c^{2} d t^{\prime 2}-d r^{\prime 2}-r^{\prime 2} d \varphi^{\prime 2}-d z^{\prime 2} \\
& r^{\prime}=\sqrt{x^{2}+y^{2}}, \varphi^{\prime}=\operatorname{arctg} \frac{y}{x}
\end{aligned}
$$

$r^{\prime}, \varphi^{\prime}-$ are polar coordinates in the plane $(x, y)$. Then we make the transformation to vortex's own frame of reference, in which each point of the vortex is at rest

$$
\begin{aligned}
& d r^{\prime}=\beta(r)\left(d r+V_{r} d t\right), \\
& d \varphi^{\prime}=d \varphi+\frac{V_{\varphi}}{r} d t \\
& t=t^{\prime}
\end{aligned}
$$

where $V_{r}$ and $V_{\phi}$ are given by the expressions [1]

$$
V_{r}=\frac{1-u}{C r^{\gamma}}, V_{\varphi}=\frac{v}{C r^{\gamma}}, \gamma=\frac{u}{1-u}
$$

For the first expression in (16) to be the total differential, we must put $\beta(r)=C_{1} V_{r}^{-1}, C_{1}$ is a constant. In its own frame of reference, the interval looks as follows

2 In the paper [1] was made an erroneous conclusion about the inapplicability of the proposed model of the relativistic Turing machine outside the critical line.

$$
\begin{aligned}
& d s^{2}=A(r)\left(c d t^{\prime \prime}\right)^{2}-d l^{2}+B(r) d r^{2} \\
& A(r)=1-\left(\frac{C_{1}}{c}\right)^{2}-\left(\frac{V_{\varphi}}{c}\right)^{2} \\
& B(r)=1-\left(\frac{C_{1}}{V_{r}}\right)^{2}-\frac{\left[\frac{C_{1}^{2}}{c V_{r}} \pm \frac{r V_{\varphi}}{c r_{\varphi}^{\prime}}\right]^{2}}{A(r)}
\end{aligned}
$$

If we put $C_{1}=c \delta / \omega, \gamma=1$ and use the previous expressions for the radial and angle components of the particle's speed (3) the formulas (18) go into the analogous formulas for the critical line (2).

From the similarity of expressions (18) and (2), we conclude that the behavior of the computation particle outside the critical line resembles its behavior on the critical line. In particular, the equations of motion of the computing particle above the horizon look the same as the equations (5) described above, with the obvious substitutions (17) instead (3). The metric under the horizon is constructed similarly to (7) with the same substitutions. The same can be said about the equations of motion of a particle under the horizon, an analog of equations (12). To solve the question of the presence (or absence) of the zeros of the $\zeta$-function outside the critical line, we must investigate the possibility of existence under the horizon the special trajectories, similar to the one whose phase portrait is depicted in Figure 2. Thus, the problem reduces to investigating solutions of the equation (analog (13))

$$
\frac{d r}{d s}= \pm \frac{C_{3}^{\prime}}{\sqrt{-A(r) B(r)}}
$$

$C_{3}^{\prime}$ is a constant, and $A$ and $B$ are given by the expressions (18), and in $B(r)\left(r_{\varphi}^{\prime}\right)^{-1}$ is set equal to zero.

According to the meaning of the calculation procedure implemented by the Turing machine, its particle-head must stop, having received the result of the calculation (if it is a matter of calculating zero) ${ }^{3}$. At the stopping point $r=0$, all the derivatives of $r$ with respect to $s$ of finite order must vanish: $r_{s}^{(n)}=0(n \geq 1$ is an arbitrary integer). Substituting in (19) the expressions for the velocities (17), we find that at $r \rightarrow 0, r_{s}^{\prime} \sim r^{\gamma}$. For the $n$-th derivative of $r$ we obtain the estimation $r_{s}^{(n)} \sim r^{n \gamma-n+1}$. In the limit $n \rightarrow \infty$, we find that the stopping condition is satisfied for $\gamma>1$ or $u>1 / 2$. Hence we conclude that in the strip $0<u<1 / 2$, the zeros of the $\zeta$-function are absent. Since the zeros of the $\zeta$-functions should be symmetrically disposed with respect to the critical line, they are absent in the strip $1 / 2<u<1$ too.

The obtained result testifies to the truthiness of the

3 If the calculated value is nonzero, then the particle, as shown above, does not stop. The result is determined by some characteristic feature of the trajectory, as described above. 
Riemann hypothesis about that all non-trivial zeroes of the $\zeta$ -function are located at the critical line

\section{Some Geometrical Aspects of the Model Proposed}

To better understand in details the motion of the computing particle and what happens at the branch points $P$ and $Q$, we do an embedding of a two-dimensional space with the metric (2) into a three-dimensional space with the Euclidean metric

$$
d L^{2}=d r^{2}+r^{2} d \varphi^{2}+d z^{2}
$$

$d L$ - is an element of length. The spatial part of the twodimensional metric (2) is written in the form

$$
r^{2} d \varphi^{2}+[1-B(r)] d r^{2}
$$

Comparing (20) and (21), we find that the metric (2) can be regarded as an induced metric on a two-dimensional surface of rotation (relative to the OZ axis) in a threedimensional Euclidean space whose equation has the form

$$
z(r)=\int^{r} \sqrt{-B(r)} d r
$$

The integral in (22) can only be determined numerically. For the calculation data shown in Figure 1, the result of calculation (22) is shown in Figure 3

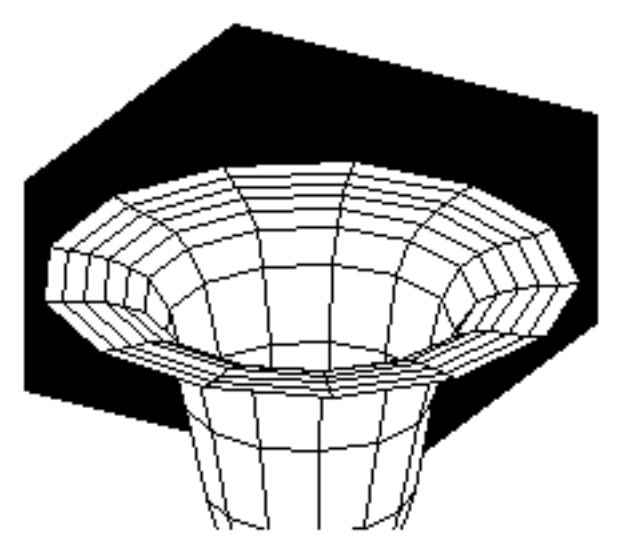

$\mathrm{X}, \mathrm{Y}, \mathrm{Z}$

Figure 3. The result of the calculation by formula (22) in the neighborhood of the point $P ; X_{m, k}=m \cos \left(\varphi_{k}\right), Y_{m, k}=\operatorname{msin}\left(\varphi_{k}\right), \varphi_{k}=2 \pi k / 13, k=1 . .13, Z_{m}=z$ $\left(r_{m}\right)$ (21). The calculation is made for the upper branch of the solution in Figure 1. The graph is non-uniformly stretched (in the upper part-larger) along the radial coordinate $r$ by replacing $r_{m}$ by $m$.

The branch points $P$ in Figure 3 are located in a circle of large radius. In order to obtain a complete surface, it is necessary to perform similar constructions for the remaining three branches-one (lower) above the horizon and two (upper and lower) below the horizon. As a result, a torus-like surface will appear on which the trajectory of the particle realizing the computation is located. The large and small torus radii (dimensionless) for the data in Figure 1 are respectively 1,33 and 0,62 . The three-dimensional trajectory of the vortex is located on the surface of the torus, passing from one part (conditionally, the upper one) to the other (lower) and vice versa at the points $P$ and $Q$.

Let's say two words about the physics of the problem under consideration. Two flat models of vortices are known in hydromechanics: a vortex-sink and a vortex-source [6]. The present examination combines the two sides of the phenomenon into single one, which corresponds to experiments with real vortices, for example, whirlpools, which have two areas of flow: convergent (surface) and divergent (in deep). In this case, the drain and source power are zero, i.e. the constituent vortices nourish each other.

\section{Discussions}

B. Riemann formulated his famous hypothesis in 1859 . So far, it has neither a clear proof nor a refutation. D. Gilbert in 1900 included it in the number of cardinal problems of mathematics under number 8. At present, the Clay Mathematic's Institute has included it among the seven problems of the millennium, for the solution of which a prize of 1 million US dollars will be paid.

A popular historical review of the problem and directions of its investigation were given in the book [7]. Most researchers of the problem believe that the Riemann hypothesis is correct, but opposite statements are also found [8]. The papers devoted to the proof of the Riemann hypothesis can be divided into works constructing proofs using purely mathematical methods [9-13] and works in which its relation to various physical problems is noted [14, 15] and accordingly using physical methods of proof.

Special mention should be made of the papers of A. Turing devoted to a numerical study of the zeros of $\zeta$-function on a classical computer. Note that Turing himself considered the Riemann hypothesis to be erroneous and his investigations were aimed at finding nontrivial zeros of the $\zeta$-function outside the critical line. A review of the papers of A. Turing is made in the report [16].

The present paper, like the previous work of the author [1, $4]$, is devoted to the calculation of the values of $\zeta$-function on a relativistic computer realizing the idea of a relativistic Turing machine.

As shown in the author's paper [17], the $\zeta$-function calculations for real arguments are connected by certain contradictions that are resolved if we assume that the geometry of the numerical continuum is different from the Euclidean one.

Note that the distinctive feature of the results obtained is the presentation of calculation as the process (as it really is) and the introduction of the concept of time, what is not typical for traditional mathematics, that claims its statements are timeless.

The question of the accuracy of relativistic computations has not yet received its final solution. The accuracy of the 
calculation of $\zeta(-1)$ obtained in [4] corresponds to an error of $3.5 \%$. If we consider this calculation as yet another confirmation of the general theory of relativity, then in accuracy it is considerably inferior to the others (experimental). As regards the accuracy of the computation of the $\zeta$-function for the complex argument presented in the present paper, from this point of view these results allow us to speak of secession of zeros from other values rather than of calculation. Therefore, the further direction of work on relativistic calculations will be an increase in their accuracy.

Let us say a few words about the derivation of the equations (12). Formally, they are obtained if we set $d \tau=0$ in the transformation formulas to the Finkelstein coordinates (7); what means the suggestion that under the horizon time "does not flow", or, more precisely, there is no very concept of time. This assumption can be justified if more deeply than is usually done, to consider the nature of phenomena under the horizon of events. As shown in the article [18], the electromagnetic vacuum below the horizon is unstable and solutions of the Maxwell equations in the form of light waves are absent. It follows that all the arguments concerning the behavior of light geodesics, given, for example in [5], and stated above, are valid only over the horizon, where the vacuum is stable. Instead of light waves (photons), the solutions of the Maxwell equations below the horizon have a tunneling character and the notion of time is inapplicable to them. They resemble the so-called "tunneling states" in a solids [19]. For the latter, the use of the concept of time and the corresponding description leads to paradoxes, for example, to an infinite speed of propagation [20].

\section{Conclusion}

In this article, a physical proof of the Riemann Hypothesis is presented.

For this, like in the previous paper of the author [1] the computation of the Riemann $\zeta$-function represented by a divergent series in the plane of the complex argument $w=u+$ $i v$ is performed using the methods of the general theory of relativity. The calculation is performed by a relativistic Turing machine, the role of the head of which is played by some material particle moving in accordance with the equations of motion found. It is shown that the noncomputable (in the sense of Turing) problem of computation of the sum of a divergent series becomes computable due to the transition to the moving system of reference.

In contrast to [1], where calculations of the $\zeta$-functions were performed on the critical line only in this article, the calculations also are made beyond the critical line. It was shown that whereas in the critical line there are two types of solutions of the relativistic equations of motion for the material particles realizing calculation that correspond to zeros and nonzero values of the $\zeta$-functions outside it, there is only one type of solution corresponding to non-zero values of the $\zeta$-function.

This gives grounds for asserting that there are no zeros of the $\zeta$-function outside the critical line, which is a proof of the Riemann hypothesis.

\section{References}

[1] Y. N. Zayko, Calculation of the Riemann Zeta-function on a Relativistic Computer, Mathematics and Computer Science, 2017; 2 (2): 20-26.

[2] G. H. Hardy, Divergent Series, Oxford, 1949.

[3] I. Nemeti, G. David, Relativistic Computers and the Turing Barrier. Applied Mathematics and Computation, 178, 118-142, 2006.

[4] Y. N. Zayko, The Geometric Interpretation of Some Mathematical Expressions Containing the Riemann $\zeta$ Function. Mathematics Letters, 2016; 2 (6): 42-46.

[5] L. D. Landau, E. M. Lifshitz, The Classical Theory of Fields, (4th ed.), Butterworth-Heinemann, 1975.

[6] L. G. Loitsyansky, Mechanics of Liquids and Gases, Moscow, Leningrad, GITTL, 1950 (Russian).

[7] John Derbyshire, Prime Obsession: Bernhard Riemann and the Greatest Unsolved Problem in Mathematics, First Plume Printing, 2004.

[8] Jinhua Fei, Riemann hypothesis is not correct, arXiv:1407. 4545v1 [math. GM] 17 Jul 2014.

[9] A. Pr'astaro, The Riemann Hypothesis Proved, arXiv:1305. $6845 \mathrm{v} 10$ [math. GM] 27 Oct 2015.

[10] R. C. McPhedran, Constructing a Proof of the Riemann Hypothesis, arXiv:1309. 5845v1 [math. NT] 30 Aug 2013.

[11] Jin Gyu Lee, The Riemann Hypothesis and the possible proof, arXiv:1402. 2822v1 [math. GM] 9 Feb 2014.

[12] V. Blinovsky, Proof of Riemann hypothesis, arXiv:1703. 03827v5 [math. GM] 1 May 2017.

[13] F. Stenger, A Proof of the Riemann Hypothesis, arXiv:1708. 01209v2 [math. GM] 14 Aug 2017.

[14] ] M. Wolf, Will a physicist prove the Riemann Hypothesis?, arXiv:1410. 1214v3 [math-ph] 1 Dec 2015.

[15] R. S. Mackay, Towards a Spectral Proof of Riemann's Hypothesis, arXiv:1708. 00440v1 [math. SP] 1 Aug 2017.

[16] Yu. V. Matiyasevich, Alan Turing and Number Theory (to the 100 anniversary of A. Turing's birth), The Alan Turing Centenary Conference (Manchester, UK, June 22-25 2012); Published in: Mathematics in Higher Education, 2012, № 10, 111-134 (Russian).

[17] Y. N. Zayko, The Second Postulate of Euclid and the Hyperbolic Geometry, arXiv:1706. 08378.

[18] Y. N. Zayko, Influence of Space-Time Curvature on the Light Propagation, SCIREA Journal of Physics, December 14, 2016, V. 1, № 1, 83-93.

[19] Y. N. Zayko, The states in the band gap of a crystal localized in the region of inhomogeneity of the external field, Solid State Physics (SU), 1976. V. 18, № 4. P. 951-955.

[20] E. O. Kane, Basic Ideas about Tunneling, in Tunneling Phenomena in Solids, Ed. by E. Burstein and S. Lundqvist, Plenum Press, NY, 1969. 